Portland State University

PDXScholar

\title{
The Vocational Interests of Prisoners: A Preliminary Validation Study of the Lustig Color Vector Test
}

\author{
Hanoch Livneh \\ Portland State University \\ Robert E. Pullo \\ University of Maine \\ Paul Lustig \\ University of Wisconsin - Madison
}

Follow this and additional works at: https://pdxscholar.library.pdx.edu/coun_fac

Part of the Experimental Analysis of Behavior Commons, and the Social Psychology Commons

Let us know how access to this document benefits you.

\section{Citation Details}

Pullo, R., Lustig, P. \& Livneh, H. (1993). The Vocational Interests of Prisoners: A Preliminary Validation Study of the Lustig Color Vector Test. Journal of Applied Rehabilitation Counseling, 24(1), 8-14.

This Article is brought to you for free and open access. It has been accepted for inclusion in Counselor Education Faculty Publications and Presentations by an authorized administrator of PDXScholar. Please contact us if we can make this document more accessible: pdxscholar@pdx.edu. 


\section{The Vocational Interests of Prisoners: A Preliminary Validation Study of the Lustig Color Vector Test}

\author{
By Robert E. Pullo \\ Paul Lustig \\ Hanoch Livneh
}

Date of A'cceptance: March 27, 1992

The purpose of this study was to provide empirical evidence for the validity of the Lustig Color Vector Test (LCVT) as a vocational interest measure. The LCVT, a nonverbal instrument, is a schematic attempt to represent the Vector Theory of Behavior which describes human behavior in terms of motion and the laws of physics. A sample of 92 participants was chosen to represent the literate male population of incarcerated offenders in the Wisconsin state prison system. Participants were administered the LCVT as well as Holland's Vocational Preference Inventory (VPI) and a demographic questionnaire. The results offered tentative support to the notion that the LCVT does measure vocational interest, thereby lending credence to Lustig's Vector Theory of Behavior. effectiveness of most verbal inventories. Finally, differing cultural backgrounds of inmates further complicate the problem. The generic issue of culture fairness is commonly a problem for all verbal inventories. From the above concerns it may be concluded that using standardized verbal inventories with many prison inmates is of limited value.

The Lustig Color Vector Test (LCVT) is a nonverbal interest and personality test which is based on vector theory. The primary purpose of the research reported here was to provide evidence of the validity of the LCVT's vectorial depiction (Lustig, 1965; Pullo, 1980) as a vocational interest measure. This was attempted by comparing the LCVT profile results with two criterion measures: expressed vocational interest as measured by a demographic questionnaire, and by the Vocational Preference Inventory (VPI), a commonly used vocational interest measure. It was hypothesized that a significant relationship would exist between responses to the LCVT and to the other two measures if indeed they all measure similar vocational interests.

\section{Vector Theory}

A close relationship exists between personality and work (Holland, 1966; Roe, 1956). For example, people who are outgoing and extroverted are believed to be suited for sales positions. However, it has often been difficult empirically to show the relationship between the two. The main problem stems from the fact that personality and work are described in two different languages. When describing personality the language takes the form of behavioral terminology such as attributes and traits, while the language of work is typically reflected in terms of functions, duties and tasks. A secondary problem is that occupations are not always clearly defined to mean the same thing in all situations. For example, a vast difference exists between the work tasks and duties of a trial lawyer and those of a brief lawyer. Lustig (1965) argues that the solution to this problem can be found in constructing a common denominator which can translate the concepts of personality and work into a common language.

Lustig attempts to solve this problem by reverting to the physical sciences and to mathematics. He states that (Lustig, Libman \& Phillips, 1978, pp. 5-6):

Work was seen as an expression of activity. Activity could be described in terms of motion. Personality, on the other hand, was seen as an expression of behavior. Behaviors 
are forms of activity. Thus work could be translated into motion and personality could be translated into motion. Both work and personality (or behavior) could be translated to same common denominator. Therefore, they were equal.
$\operatorname{Activity}(\mathrm{A})=$ Motion (M)
$A=M$
Behavior $(B)=\operatorname{Activity}(A)$ or Motion $(M) B=A$ or $M$
Work $(W)=$ Activity $(A)$ or Motion $(M) W=A$ or $M$
Behavior $(B)=$ Work $(W)$
Personality $(P)=$ Behavior $(B)$
Work $(W)=$ Behavior $(B)$
Personality $(\mathrm{P})=$ Work $(\mathrm{W})$
$\mathrm{B}=\mathrm{W}$
$\mathrm{P}=\mathrm{B}$
$\mathrm{W}=\mathrm{B}$
$\mathrm{P}=\mathrm{W}$

Though both personality and work have other attributes such as purpose and volition, the essence is that of adjustment within the environment: activity or motion. Thus, Lustig arrives at a second basic assumption that:

... matter and motion are inseparable. One cannot have matter without motion, one cannot have motion without matter. The proposition is that behavior is activity which in turn is motion. Any form of behavior can be viewed as reflecting a change in position. Something is in motion. Thus, in order to examine behavior, one needs to relate it to motion. The problem now is to examine the nature of motion. Later the nature of motion will be translated into human behavior (Lustig, Libman \& Phillips, 1978, p. 6).

Lustig states that all motion consists of several basic elements. These elements include: (1) What - the substance or matter which is in motion; (2) Where - the location of the start and the end of the motion (source and direction); (3) When - the duration of time during which the motion takes place and the rate of the movement; (4) How much - the amount of force of the motion; (5) How - the types of motion that occur; and (6) Why - the purpose or reason for motion to begin and end.

Since Lustig equates behavior with motion, he now can describe behavior as an aspect of motion, using the same elements for motion as previously stated (Lustig, Libman \& Phillips, 1978, p. 6).

1. Substance or materials of behavior are motoric, emotional, and intellectual. There may be other behavioral substances such as extrasensory perception, intuition, etc., but their existence is still regarded as doubtful and are therefore omitted at this time.

2. Sources of behavior refer to the location of the initiating stimulus that causes movement. These sources of behavior or initiating stimuli are either internal to the person, external or both.

3. Direction of behavior is concerned with the notion that behavior exerted toward a certain location or object with respect to the person. The two main directions of behavior are flexor or moving toward the person, and extensor or moving away from the person. There may also be another type of motion which can be described us circular. In this instance, the direction of the behavior is described as extensor-flexor.

4. Time of behavior refers to the duration of the activity. Here the time can vary from extremely short to extremely long in duration.

5.Speed of behavior refers to the rate or pace of behavior. Frequently, the concept of temperament is used to describe the speed or rate of behavior. Speed of behavior can range from extremely slow to extremely fast.

6. Force of behavior refers to the strength or impact of the behavior. Force can range from extremely weak to extremely strong.

7. Purpose of behavior or motion (i.e., why) relates to the concept of imbalance of forces and/or internal need to act and move. Imbalance of forces is concerned with homeostasis, while internal need to act or move is probably concerned with biological or instinctual drives to act.

Lustig (1977) later added another component, permeability. Permeability, or penetrability, is described as the person's capacity to accept or be stimulated by the behavior or motion from the environment and to maintain his or her own integrity and identity. Permeability may range from very weak or highly permeable, to very strong or highly impermeable.

The reasoning that guided Lustig in evaluating the substance or material components of behavior stemmed particularly from earlier work on the psychology of color (see, for example, Luscher, 1969; Schaie, 1963). According to this reasoning the colors, yellow and red, pertain to the emotional dimension of human behavior. These colors, therefore, more directly reflect interpersonal relations or focus on people. The colors, blue and green, relate to the motoric or physical aspects of human functioning. As such, these two colors appear to focus on "things" or inanimate environmental objects. Finally, the third dimension is composed of the colors, white and black. This behavioral component reflects the intellectual sphere. In other words, it is more directly related to ideational or cognitive functioning. Despite the conceptual appeal of this theoretical model, the scoring system for measuring these three behavioral elements is not finalized as of yet, and therefore, the validity of this behavioral component was not addressed in the present study.

All behavior is theoretically represented and accounted for in the above components. Lustig sees the converse as equally valid: that all behavior should be capable of description in terms of the above elements. "In short, it is the beginnings of a Vector Theory of Behavior" (Lustig, Libman \& Phillips, 1978, p. 9).

\section{The Lustig Color Vector Test}

The Lustig Color Vector Test (LCVT) is composed of 96 cards which schematically represent vector theory. Each card $(82 \mathrm{~mm} \times 82 \mathrm{~mm})$ contains a printed square $(41 \mathrm{~mm} \times 41 \mathrm{~mm})$ and arrows. Each card differs from the others on the basis of the following:

1) Source--Internal vs. External;

2) Direction--Flexor vs. Extensor;

3) Color--Yellow, Red, Blue, Green, White, and Black; 


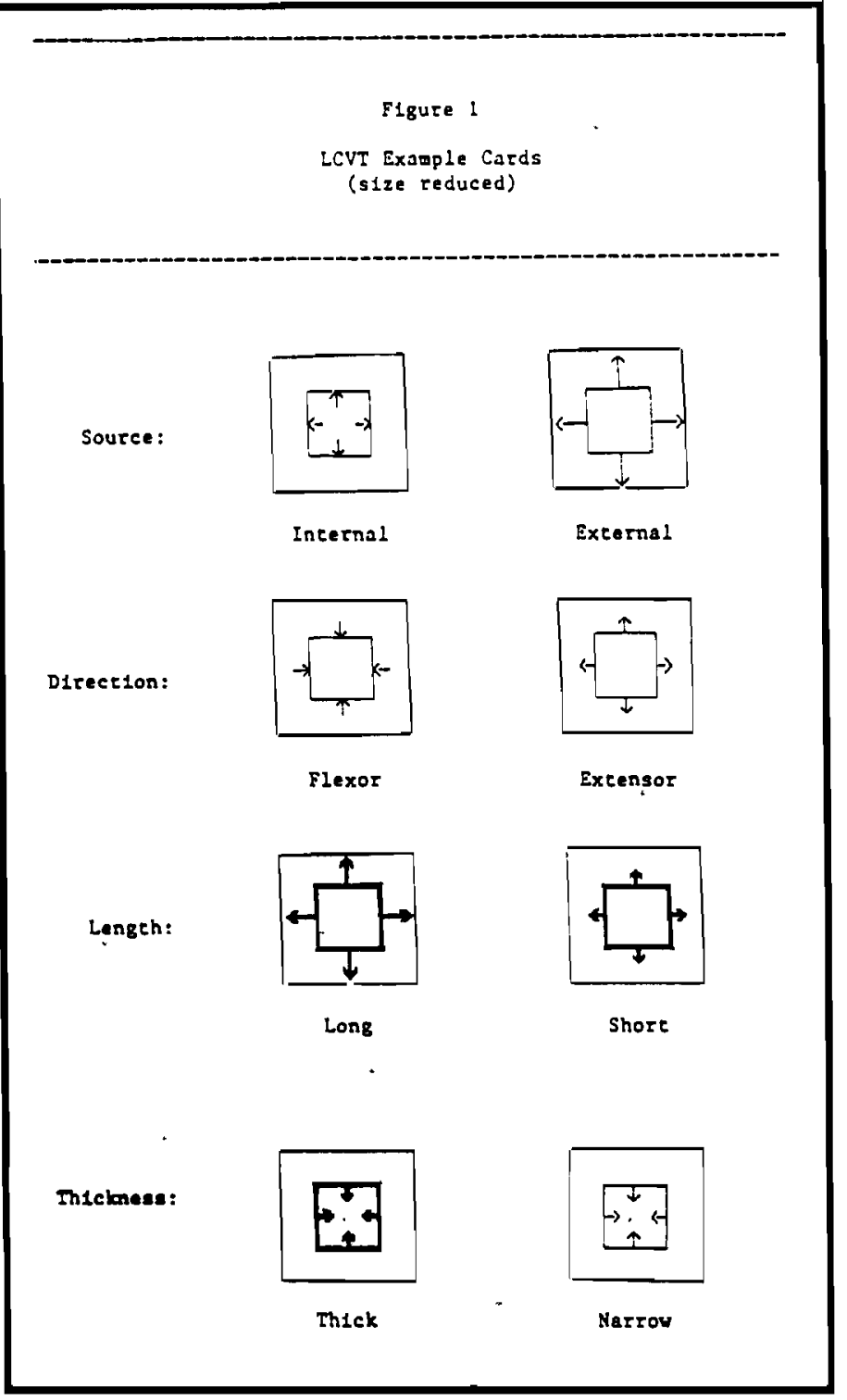

4) Arrow Length- Short (10.5 mm) vs. Long (21.0 mm);

5) Thickness--Narrow $(.5 \mathrm{~mm})$ vs. Thick $(4.0 \mathrm{~mm})$; (see Figure 1 for examples)

Thus, the test contains the following total number of representations: Source (2) X Direction (2) X Color (6) X Length (2) $X$ Thickness (2) $=96$.

In administering the test, the entire set of cards is given to the subject who is asked to select the 24 cards "most liked" and the 24 cards "least liked".

Lustig (Lustig, Libman \& Phillips, 1978) makes the following assumptions regarding the LCVT:

1. The 96 cards describe almost all of human behavior,

2. Any human event or behavior is some combination of the color-vector cards.

3. The number of events and/or behaviors that a set can describe is 96 ! (96x $95 \times 94 \times 93 . .$.

4. Those factors omitted in each set of cards are seen as less significant than those included.
5. The design is more objective than the words used to describe it.

6. Each card is represented in the same way to all people.

7. People who indicate their likes and dislikes are responding in the same way.

8. Choices are valid expressions of one's likes and dislikes.

The scoring system is additive. The test is scored independently according to vectors derived from the sources and directions of the test card arrows. For example, if a subject chooses as "likes": three A's, five B's, thirteen C's, and three D's; and "dislikes": eight A's, six B's, zero C's, and ten D's, the score is computed as follows (Livneh, 1976):

$$
\begin{aligned}
& \text { Vector } A=A_{i} \text { Likes }+\left(-A_{i} \text { Dislikes }\right)=3+(-8)=-5 \\
& \text { Vector } B=B_{i} \text { Likes }+\left(-B_{i} \text { Dislikes }\right)=5+(-6)=-1 \\
& \text { Vector } C=C_{i} \text { Likes }+\left(-C_{i} \text { Dislikes }\right)=13+(0)=+13 \\
& \text { Vector } D=D_{i} \text { Likes }+\left(-D_{i} \text { Dislikes }\right)=3+(-10)=-7
\end{aligned}
$$

$\begin{array}{llllll} & \text { A } & \text { B } & \text { C } & \text { D } & \\ \text { Likes (+) } & 3 & 5 & 13 & 3 & =24 \\ \text { Dislikes (-) } & 8 & 6 & 0 & 10=24 \\ & \frac{-5}{-1} & \frac{1}{13} & \overline{-7} & \end{array}$

${ }^{1}$ Note: Because each pile contains an equal number of cards (24), summing all the vectors together will result in a final score of zero. Thus, $(-5)+(-1)+(+13)+(-7)=0$. In determining vector orientation it is important to remember that, according to vector theory, vectors $A$ and $C$ and vectors $B$ and $D$ are geometric opposites, respectively. Therefore, a negative value of one is equal to a positive value of the other. Thus,

$$
\begin{aligned}
& A \cdot C=(-5)-(13)=-18 A \text { or } 18 C \\
& B-D=(-1)-(-7)=6 B \text { or }-6 D \\
& \text { or } \\
& \begin{array}{lllll}
-5 \mathrm{~A} & =+5 \mathrm{C} & \text { and }+7 \mathrm{~B} & =-7 \mathrm{D} \\
-13 \mathrm{~A} & =+13 \mathrm{C} & & -1 \mathrm{~B} & =+1 \mathrm{D} \\
\overline{-18 \mathrm{~A}} & \overline{+18 \mathrm{C}} & \overline{+6 \mathrm{~B}} & \overline{-6 \mathrm{D}}
\end{array}
\end{aligned}
$$

Presented graphically, Figure 2 shows the resultant vector orientation:

Note that $C=-A$, and $D=-B$ (i.e., movement towards $A$ is also movement away from $C$, and movement towards $B$ is also. movement away from $D$.

In this example the subject's resultant vector orientation falls' in the CB area.

\section{Vector Theory and the World of Work}

Roe (1956) divided the world of work into eight vocational areas and six levels within each area. Roe's eight areas are very' similar to the eight areas in Lustig's vector theory. Figure 3 sum marizes Roe's configuration.

Holland $(1966,1973)$ also has a closely allied theory of the world of work, which is composed of six different environments, Holland equates interest tests with personality tests and there fore divides personality into six similar types. He places the six types of personality on a hexagon, with each type in a particular 
Figure 2

Resuleane Vector Orientacion

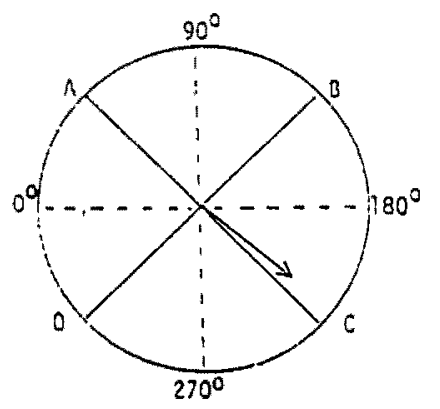

Figure 3

The World of Work according to Roe

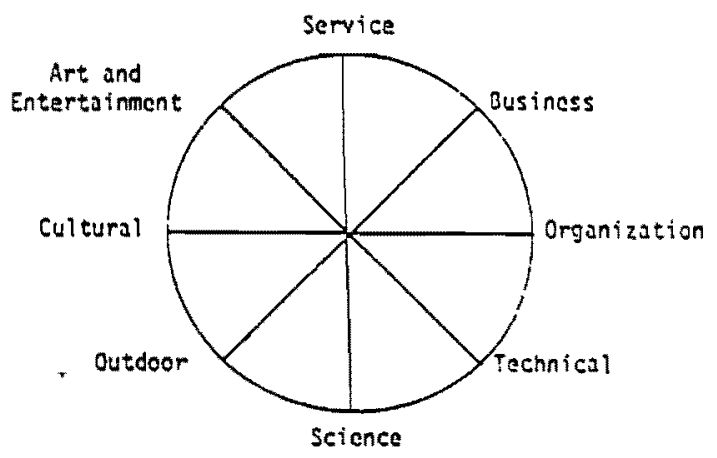

The differences in the conceptualizations of Roe, Holland and Lustig lay in the changes in position and titles of the vocational areas (Pullo, 1980). Lustig (1965) positions the vocational areas according to the relationship of orientations $(A, B, C, D)$.
Figre 4

The World of Work aceording to Holland

Soctal" Enterpeising

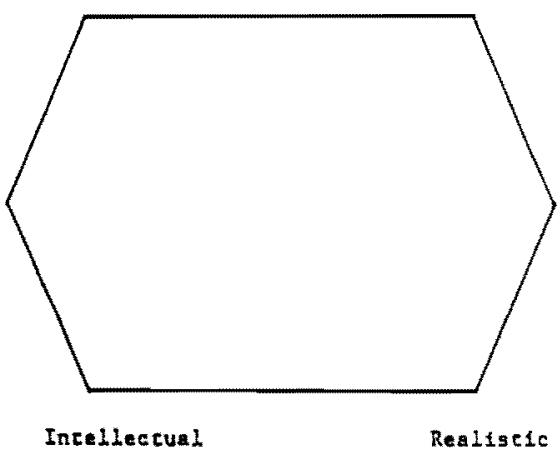

Figure 5 demonstrates Lustig's conceptualization of the positions of the eight vocational areas in relationship to the locations of the four vector orientations $(A, B, C, D)$.

Figure 5

The World of work according to Lustig.

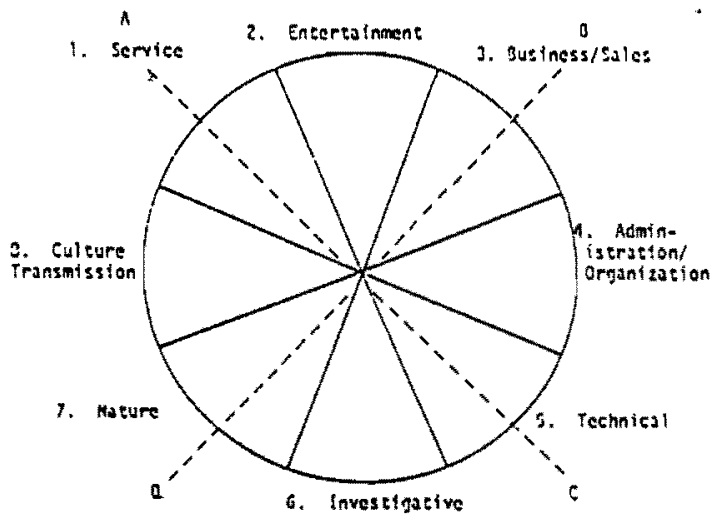

Method

\section{Sample}

A total of 117 subjects were referred by prison administration for group and individual testing during a five week period. Of this group seven subjects chose not to participate and eighteen additional subjects were eliminated due to missing data or invalid profiles. The final sample consisted of 92 subjects: 52 from 
the Waupun Correctional Institution (WPI) and 40 from the Green Bay Correctional Institution (GBCI). These subjects were all male, predominantly white $(65.2 \%)$, and unmarried $(80.9 \%)$. The ages of the participants ranged from 17 to 73 , with a mean age of 26.9 years, and a standard deviation of 9.1 . Years of schooling ranged from 5 to 16 years with a mean of 10.81 years and a standard deviation of 1.75. Table 1 contains the expressed vocational interest results of the participants.

Table 1

Expressed Vocational Interests of Study Participants
Areas

1. Service

2. Entertainment

3. Business/Sales

4. Administrative/Organizational

5. Technical

6. Investigative

7. Nature

8. Culture Transmission
Percentages

$9.4 \%$

$9.4 \%$

$8.2 \%$

$5.9 \%$

$44.7 \%$

$0 \%$

$18.8 \%$

$3.5 \%$

\section{Procedure.}

Testing, in groups of 8 to 20 persons, was supervised by the senior author. Participants were tested in well-lighted and comfortable classrooms at each institution. All participants were uniformly administered a demographic data questionnaire, the Vocational Preference Inventory (VPI), and the Lustig Color Vector Test in a single sitting.

\section{Statistical Analysis}

The primary methods of data analysis were complete link clustering (Johnson, 1967; McQuitty, 1960) and quadratic assignment (Baker \& Hubert, 1977; Baker, Hubert \& Schultz, 1977; Hubert \& Schultz, 1976).

Complete link clustering was used to empirically identify clusters of individuals who were similar with respect to their profiles of scores on the LCVT and on the VPI. The clustering solution produced a dendogram which revealed the hierarchical grouping process, as well as the diameter (i.e., dissimilarity) values for each partition level in the hierarchical grouping process. Each set of diameter values was then plotted with the number of clusters corresponding to each partition level. An inspection of the plot revealed that an "elbow" in the diameter values appeared at a particular partition level, signifying that within-group similarity dropped off considerably in proceeding to the next partition. The iteration level where this "elbow" occurred was then chosen as the "optimal" partition in the hierarchy. The same procedure was also used for the clustering of VPI subject profiles in order to achieve the selection of parsimonious and homogeneous clusters of subjects.
Once the clustering procedures were complete, the quadratic assignment method was used to determine the similarity of the LCVT clustering with the VPI clustering. The quadratic assignment procedure was used to test the similarity of the clusters on the LCVT, the VPI, and the respondents' expressed choice, which was determined from a list of 41 descriptors listed on the demographic data questionnaire. The descriptors were chosen, based on task and role, to represent the eight work clusters consistent with Lustig's vector theory.

A non-parametric technique in the static mode, the aim of quadratic assignment is to test an hypothesis regarding whether an observed proximity matrix (Q) reflects an hypothesized structure matrix $(\mathrm{C})$, thus confirming that the $\mathrm{C}$ matrix underlies the $Q$ matrix for a given reorganization of the $Q$ matrix (Baker \& Hubert, 1977). The Q matrix is some measure of pairwise association of the observed data (i.e., LCVT clusters). The $\mathrm{C}$ matrix constitutes an hypothesized configuration of the pairwise association between these variables (i.e., VPI or expressed interest clusters).

A measure of correspondence, gamma ( $r$ ), is then computed between the two matrices. In order to evaluate the size of gamma, a null hypothesis is invoked to obtain a reference distribution using a simple variant of the randomization model of non-parametric statistics (Baker \& Hubert, 1977; Bradley, 1968).

The quadratic assignment program of Baker, Hubert \& Schultz (1977) was used to compare the various profile clusterings. The null hypothesis is typically rejected if the $Z$ value corresponding to the obtained gamma index is greater than or equal to 1.65. This would provide for a one-tailed test at the a $=.05$ level.

\section{Results and Discussion}

\section{Clustering Results}

Two sets of LCVT scores were compared with the two vocational preference criterion measures, namely LCVT AB and LCVT 1-8. The LCVT AB scores were simply the two numbers which represent the scoring along the A-C continuum and the $B-D$ continuum. For the LCVT 1-8 scores, the "A" and "B" orientation on the LCVT profiles were plotted according to Lustig's theory to determine the area of vocational interest (see Figure 5).

Thus the subjects were categorically clustered according to one of the eight vocational areas into which the resultant vector fell (Table 2). The results from Table 3 indicate the level of correspondence between the various LCVT profile clusterings and the respective criterion clusterings.

The results of this study lend only partial support to the LCVT's construct validity. It appears that the vectorial repre: sentation of the test as depicted in the four-vector system (i.e. A through D), was partially supported (see two top rows of Table 3). The LCVT vector orientations were significantly as. sociated with participants' expressed interests $(\mathbf{r}=.56 ; \mathbf{p}=$ .0007 ), while approaching significance with the VPI profile (1 $=.31 ; \mathbf{p}=.06)$. On the other hand, the LCVT eight are: categorical representation failed to correspond significantl. 
Table 2

Resultant Vector Areas of LCVT 1-8 Profile Results

Area

1. Service

2. Entertainment

3. Business/Sales

4. Administration/Organization

5. Technical

6. Investigative

7. Nature

8. Culture Transmission

9. Missing Cases
\# of subjects $\%$ of subjects

$6 \quad 6.52$

$9 \quad 9.78$

$10 \quad 10.87$

$15 \quad 16.30$

$11 \quad 11.96$

$27 \quad 29.34$

$11 \quad 11.96$

$2 \quad 2.17$

$1 \quad 1.09$

ture," may require the least amount of knowledge or education, while the two least chosen areas, "Investigative" and "Culture Transmission," often require the most knowledge, education or training. The difficulty in describing a group with ill-defined interests is caused by the fact that a short resultant vector is very changeable, or weaker, since it is composed of a larger number of forces. On the other hand, a longer vector is less changeable, or stronger, hence it is better defined.

Another finding of interest in this study was the non-significant relationship between the LCVT 1-8 area representation and respondents' expressed interest. This, coupled with the significant relationship between the LCVT $A B$ vectorial results and the directly expressed interests, leads one to question the validity of the plotting of the resultant vector. Since the plotting of the "A" and "B" orientations on the vocational interest circle is a simple transformation of two variables into a combined variâble, it was theorized that this third

Table 3

Quadratic Assignment Results of Comparison of Profile Clusterings

\begin{tabular}{|c|c|c|c|c|c|}
\hline Q Matrix & C Matrix & $\underset{(\mathbf{r})}{\text { Gamma }}$ & $\begin{array}{c}\mathbf{Z} \\
\text { Score }\end{array}$ & Result & $\begin{array}{c}\text { Probability } \\
\text { Level }\end{array}$ \\
\hline \multirow{2}{*}{ LCVT AB } & VPI 6 & .31 & .20 & NS & \\
\hline & Exp. Interest & .56 & 3.22 & Sign. & .0007 \\
\hline \multirow[t]{2}{*}{ LCVT $1-8$} & VPI 6 & .20 & -1.23 & NS & \\
\hline & Exp. Interest & .27 & -.65 & NS & \\
\hline VPI 6 & Exp. Interest & .43 & 3.15 & Sign. & .0007 \\
\hline
\end{tabular}

with the VPI 6 occupational areas. A possible explanation for the lack of correspondence between the two models may be that although Lustig's theory and Holland's theory are quite similar, the instruments derived from their theories, the LCVT and the VPI, do not produce similar results. In other words, their psychometric properties (e.g., scoring systems) are rather different.

Also, although the two models use similar terminology which suggests certain equality they may, in fact, be dealing with different conceptualizations of the world of work and of personality. Suppose that theory $A$ is concerned with what is happening and theory $B$ is concerned with why it is happening. Metaphorically, it may be that one is concerned with fruit in terms of roundness, color and size (VPI), while the other is dealing with fruit in terms of vitamins and sugar content (LCVT). Similarly, there are different ways of looking at people's behavior. The VPI deals with "what" (events), while the LCVT deals with "Why" (ways of dealing with the self and the world around it).

Although the LCVT's AB vectorial depiction demonstrated a significant relationship with respondents' expressed interest, the individual LCVT AB clusters were difficult to decipher. There was considerable inconsistency in terms of expressed interest. It is likely that the nature of the sample may be the primary cause for this inconsistency. The participants in the sample most likely had limited knowledge of the world of work as well as limited work experience. This is consistent with the fact that the two dominant interest areas, "Technical" and "Na- variable, the resultant vector, would, in turn, reflect the vocational interests of the respondents in similar groupings. This, however, did not occur in the latter case. The two data sets, the LCVT 1-8 and the expressed interests, did not significantly correspond with each other.

There are several explanations for this lack of correspondence. First, and most obvious, is the possibility that the plotting of the resultant vector is psychometrically incorrect. This would involve a major reworking of Lustig's scoring system itself, at least in regard țo the resultant vector plot.

A second explanation, and probably the more likely one, refers to the nature of the sample and the meaningfulness of the LCVT AB clusters. It should be recalled that this sample had relatively little work experience and/or knowledge of the world of work. In examining the nature of the clustering solution, the researchers observed a large number (38) of participants in cluster 1 . Since $50 \%$ of these subjects had chosen "Technical" as their vocational interest area, it was assumed that this cluster reflects interests in occupations such as technician, repairman, auto mechanic, and factory worker. However, further inspection of this cluster also demonstrated it to have the shortest mean resultant vector: its " $A$ " and "B" orientations were extremely close to zero. In essence, then, this short vector could indicate vocational interests which are not clearly defined.

If one accepts cluster 1 as composed of those participants who have ill-defined vocational interests, one may infer that the subjects in cluster 1 tended to choose their LCVT cards more randomly that the other subjects. If this is indeed so, the non- 
significant result between the LCVT 1-8 and expressed interests would naturally follow. Therefore, it is likely that the present group of respondents, due to their vocational limitations, constituted a biased sample and this, in turn, had a deleterious effect on their choice of cards (e.g., only weak or unstable preferences).

The result, then, was less meaningful (e.g., shorter) resultant vectors, which limited vector score variability and, hence, the correlation coefficients between the LCVT scoring system and the directly, expressed interests. Furthermore, the latter may also have unrealistic interests due to the participants' lack of vocational experience and the yet to be served prison terms. In the present study only the vectorial depiction of the LCVT was studied in its relationship to vocational interest. Inasmuch as the theory underlying the LCVT also regards the factors of color, arrow and square thickness, among others, to be of importance in occupational choice, these were not addressed in the present study. Hence, the depiction of the eight occupational areas as relying mainly on the length of vectorial representation may have been an oversimplification of Lustig's theory, and the failure to support a linkage between the directly expressed interests and the octant representation may be partially due to this oversight.

In summary, if the present results are regarded as nonspurious, the non-significant correspondence between the LCVT 1-8 and the respondents' expressed interests may be better explained as a result of limited vocational interest or experience by a large number of participants. Though no data were gathered on the participants' work experience, the mean age of 26.9 years and the mean number of school years of $\mathbf{1 0 . 8}$ certainly indicate that the respondents' work experience was quite limited. Future research with subject populations with clearly defined vocational interests and more extensive occupational experience could be of value in clarifying this issue.

\section{Conclusion}

The purpose of this study was to provide preliminary data on the validity of the Lustig Color Vector Test. The fact that certain LCVT subscores seem to reflect vocational interests manifested by participants lends some credence to Lustig's vector theory of behavior. Further research on the LCVT's scoring system may suggest future-modification of its present administration procedures (i.e., choice of 24 most liked and disliked cards), scoring system, and perhaps of the test itself. It may also shed light on other personality aspects and their inner dynamics.

References

Baker, F. \& Hubert, L. (1977). Data analysis: A unified ap- proach. Occasional Paper No. 13. Madison, WI: University of Wisconsin.

Baker, F., Hubert, L. \& Schultz. J. (1977). Quadratic assignment program. University of Wisconsin: Laboratory of Experimental Design.

Bradley, J. (1968). Distribution-free statistical tests. Englewood Cliffs, NJ: Prentice-Hall.

Glaser, D. (1964). The effectiveness of a prison and parole system. NY: Bobbs-Merrill.

Glaser, D. (1966). The public offender as client. Rehabilitation Record, Sept.-Oct., 17-19.

Holland, J. (1966). The psychology of vocational choice. Lexington, MA: Blarsdell.

Holland, J. (1973). Making vocational choices. Englewood Cliffs, NJ: Prentice-Hall.

Hubert, L. \& Schultz, J. (1976). Quadratic assignment as a general data analysis technique. British Joumal of Mathematics and Statistical Psychology, 29, 87-110.

Johnson, S. (1967). Hierarchical clustering themes. Psychometrika, 32, 241-254.

Livneh, H. (1976). The CVT and its relationship to disability type and people's reactions. Unpublished doctoral dissertation. University of Wisconsin - Madison.

Luscher, M. (1969). The Luscher Color Test. New York: Random House.

Lustig, P. (1965). The Color-Vector Test. Unpublished manuscript. University of Wisconsin - Madison.

Lustig, P. (1977). Studies with the color-vector cards. Unpublished manuscript. University of Wisconsin - Madison.

Lustig, P. Libman, L., \& Phillips, R. (1978). The use of the Lustig Color-Vector Test in rehabilitation. University of Wisconsin -Madison.

McQuitty, L. (1960). Hierarchical linkage analysis for the isolation of types. Educational and Psychological Measurement, 20, 55-67.

Pullo, R. (1980). A validation of the Lustig Color Vector Test in an offender population. Unpublished doctoral dissertation. University of Wisconsin - Madison.

Roe, A. (1956). The psychology of occupations. New York: John Wiley \& Sons.

Schaie, K.W. (1963). The Color Pyramid Test: A nonverbal technique for personality assessment. Psychological Bulletin, $60,530-547$.

Wilkins, L. (1969). Evaluation of penal measures. New York: Random House.

Robert E. Pullo, Ph.D., CRC is Associate Professor and Chairperson, Department of Health and Rehabilitation, University of Maine, Farmington, Maine.

Paul Lustig, Ph.D., at the time of his death, was retired as Professor, Department of Rehabilitation Psychology and Special Education, University of Wisconsin, Madison, Wisconsin.

Hanoch Livneh, Ph.D., CRC is Associate Professor and Coordinator, Rehabilitation Counseling Specialization, Portland State University, Portland, Oregon. 